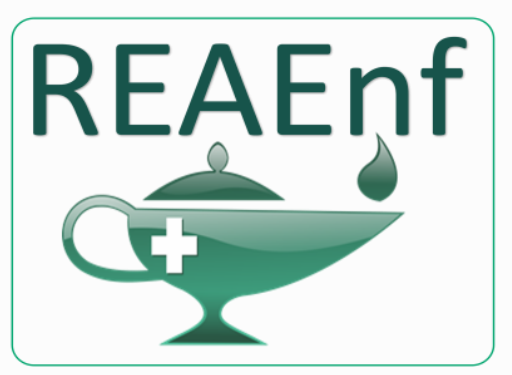

Revista Eletrônica Acervo Enfermagem
ARTIGO ORIGINAL

Recebido em: 11/2019

Aceito em: $12 / 2019$

Publicado em: $3 / 2020$

\title{
Desfechos obstétricos em gestações tardias no estado de Minas Gerais
}

\author{
Obstetric outcomes in late pregnancies in the state of Minas Gerais
}

\section{Resultados obstétricos en embarazos tardíos en el estado de Minas Gerais}

Daniela Nogueira de Oliveira ${ }^{1 *}$, Jonathan Mendes de Castro1 ${ }^{1}$ Talles Vinícius de Castro Oliveira1, Enaile de Souza Proti ${ }^{1}$, Ranyelli Estefany da Costa Martins ${ }^{1}$, Marcela Alves Azevedo ${ }^{1}$, Diego Azevedo Araújo ${ }^{1}$, Jacqueline Souza Dutra Arruda ${ }^{1}$, Carlos Henrique Wernersbach Guerra ${ }^{1}$, Wendel Jose Teixeira Costa ${ }^{1,2}$.

\begin{abstract}
Resumo: Essa pesquisa teve por objetivo descrever os desfechos obstétricos das gestações tardias no Estado de Minas Gerais, no período de 2013 a 2017. Trata-se de estudo transversal descritivo, com utilização dados secundários provenientes do Sistema de Informação sobre Nascidos Vivos (SINASC) do Departamento de Informática do Sistema Único de Saúde (DATASUS). A população do estudo foi composta por um total de 1.108.615 nascidos vivos residentes no Estado de Minas Gerais, no período analisado. Desses, 916.251 (82,6\%) nasceram de mulheres com idade igual ou superior a 35 anos e $192.364(17,4 \%)$ de mulheres com idade até 34 anos. A análise dos desfechos obstétricos na gestação tardia demonstrou maior ocorrência de gestação pré-termo $R R=1,25$ (IC95\% 1,23-1,26), com número consultas pré-natal de 7 ou mais consultas $R R=1,04$ (IC95\% 1,04-1,04), parto cesáreo $R R=1,20$ (IC95\% 1,19-1,20), índice de Apgar até 7 no $1^{\circ}$ minuto $R R=1,08$ (IC95\% 1,07-1,10), e no $5^{\circ}$ minuto $R R=1,20$ (IC95\% 1,16-1,24), baixo peso ao nascer $R R=1,28$ (IC95\% 1,26-1,29) seguido por alto peso $R R=1,14($ IC95\% 1,11-1,17) e anomalias congênitas $R R=1,40$ (IC95\% 1,33-1,48), ambas com associação estatística significativa.
\end{abstract}

Palavras-chave: Gravidez, Idade materna, Perfil de saúde.

Abstract: This research aimed to describe the obstetric outcomes of late pregnancies in the state of Minas Gerais, from 2013 to 2017. This is a descriptive cross-sectional study using secondary data from the Live Birth Information System (SINASC). Department of Informatics of the Unified Health System (DATASUS). The study population consisted of a total of 1,108,615 live births residing in the State of Minas Gerais during the analyzed period. Of these, 916,251 (82.6\%) were born to women aged 35 and over and 192,364 (17.4\%) to women aged 34 and older. The analysis of obstetric outcomes in late pregnancy showed a higher occurrence of preterm pregnancy $\mathrm{RR}=1.25(95 \% \mathrm{Cl} 1.23-1.26)$, with a number of prenatal consultations of 7 or more $\mathrm{RR}=1.04(95 \% \mathrm{Cl} 1.04-1.04)$, cesarean delivery $\mathrm{RR}=1.20(95 \% \mathrm{Cl} 1.19-1.20)$, Apgar score up to 7 in the 1 st minute $\mathrm{RR}=1.08(95 \% \mathrm{Cl} 1.07-1.10)$, and at 5 minutes $\mathrm{RR}=1.20(95 \% \mathrm{Cl} 1.16-1.24)$, low birth weight $\mathrm{RR}=1.28(95 \% \mathrm{Cl} 1.26-1.29)$ followed by high weight $\mathrm{RR}=1.14(95 \% \mathrm{Cl} 1.11-1.17)$ and congenital anomalies $\mathrm{RR}=1.40(95 \% \mathrm{Cl} 1.33-1.48)$, both with significant statistical association.

Keywords: Pregnancy, Maternal age, Health profile.

Resumen: Esta investigación tuvo como objetivo describir los resultados obstétricos de embarazos tardíos en el estado de Minas Gerais, de 2013 a 2017. Este es un estudio descriptivo de corte transversal que

${ }_{1}^{1}$ Centro Universitário de Caratinga (UNEC), Caratinga-MG. *E-mail: daninog2301@hotmail.com

2 Laboratório de Escrita Científica, Escola Superior de Ciências da Santa Casa de Misericórdia (EMESCAM), Vitória-ES. 
utiliza datos secundarios del Sistema de Información de Nacimientos Vivos (SINASC) del Departamento de Informática del Sistema Único de Salud (DATASUS). La población de estudio consistió en un total de $1,108,615$ nacidos vivos que residían en el estado de Minas Gerais durante el período analizado. De estos, 916,251 (82.6\%) nacieron de mujeres mayores de 35 años y $192,364(17.4 \%)$ de mujeres mayores de 34 años. El análisis de los resultados obstétricos al final del embarazo mostró una mayor incidencia de embarazo pretérmino $\mathrm{RR}=1.25$ (IC 95\% 1.23-1.26), con varias consultas prenatales de 7 o más $\mathrm{RR}=1.04$ ( IC 95\% 1.04-1.04), cesárea RR $=1.20$ (IC 95\% 1.19-1.20), puntaje Apgar de hasta 7 en el primer minuto $R R=1.08$ (IC 95\% 1.07-1.10), y a los 5 minutos $R R=1.20$ (IC 95\% 1.16-1.24), $R R$ de bajo peso al nacer $=$ 1.28 (IC 95\% 1.26-1.29) seguido de alto peso $R R=1.14$ (IC 95\% 1.11-1.17) y anomalías congénitas $R R=$ 1.40 (IC 95\% 1.33-1.48), ambos con asociación estadística significativa.

Palabras clave: Embarazo, Edad materna, Perfil de salud.

\section{INTRODUÇÃO}

Gravidez é um fenômeno fisiológico decorrente da fecundação do óvulo pelo espermatozoide. Geralmente ocorre dentro do útero da mulher, primeiramente ocorre a formação do zigoto e posteriormente a formação embrionária e assim após a oitava semana o desenvolvimento fetal. O período gestacional da mulher traz consigo transformações fisiológicas, físicas no corpo da mulher, assim como emocionais, econômicas e sociais, consequentemente o número de mulheres atualmente que vem adiando esse acontecimento e preferenciando uma gestação tardia aumentou significativamente na última década (MALDONADO MT, 2002; TSUNECHIRO MA e BONADIO IC, 1999).

Essa tendência crescente de adiar a gravidez têm sido observada especialmente entre mulheres de países desenvolvidos (MATHEWS TJ e HAMILTON BE, 2002). Com a modificação do espaço da mulher nas atividades cotidianas, a escala de prioridade em relação a trabalho, relacionamento conjugal $\mathrm{e}$ sociedade passaram a ter prioridades diferentes comparadas com o último século. A escolha pela maternidade também passou a se modificar com esse novo cenário e a gravidez na idade tardia vem ocorrendo com uma maior frequência na vida da mulher (SENESI LG, et al., 2004).

Algumas mudanças já são observadas no comportamento da mulher nos tempos atuais no que diz respeito a maternidade. Optar por ter menos filhos é uma dessas mudanças, assim como a de adiar a gravidez. Adiar uma gestação decorre em muitas das vezes da tomada de decisões como priorizar uma formação e se estabelecer e se equilibrar financeiramente primeiro, mas isso não significa que essa mulher não deseja ser mãe. A maternidade é muito valorizada, desde os tempos antigos, vista até como uma necessidade intrínseca da mulher. Culturalmente, muitos povos enalteciam a procriação, a ponto de haver tratamento diferenciado à mulheres férteis para com as inférteis. Assim como também existe a visão cultural da infertilidade ser um problema único da mulher, conquanto a fertilidade masculina é a última a ser investigada, e por muita das vezes nem ser levada em consideração. A maternidade ainda é para muitas mulheres uma meta de vida. $E$ assim, fazem a escolha de serem mães mais velhas (TANAKA ACA $e$ ALVARENGA AT, 1999).

Com o avanço da tecnologia, a reprodução assistida, oferecida a mulheres com infertilidade involuntária tem apresentado altas taxas de sucesso, aumentando assim, também, o número de mulheres mais velhas gestantes. Porém, gestações em idade mais avançadas apresentam maior risco de complicações, podendo a gestação tardia ser uma gestação de alto risco (ZIADEH SM, 2002).

A gestação tardia é aquela que ocorre após os 35 anos ou mais de idade. Sendo considerada mais arriscada por ser uma gravidez onde a mulher já está em processo de declínio da saúde e não desfruta da mesma quando comparado a saúde de uma mulher mais jovem (ANDRADE PC, et al., 2004). Nos Estados Unidos, as taxas de natalidade entre mulheres acima de 35 anos de idade aumentaram progressivamente $\mathrm{e}$ atingiram seu nível mais elevado em 2006, com 47,3 (35 a 39 anos) e 9,4 (40 a 44 anos) nascimentos por 1.000 mulheres, respectivamente (MARTIN JA, et al., 2008). Na Noruega, tendência semelhante foi observada, onde a proporção de gestações tardias dobrara nos últimos 30 anos (TANBO T, 2008). 
No ano de 2012 o total de partos em mulheres acima de 35 anos no Brasil representaram $11,28 \%$ sendo que a região sudeste teve o maior índice de partos representando $13,26 \%$. No período de 2006 a 2012 a proporção em gestação em idade avançada aumentou $18,1 \%$, entretanto o número de nascidos vivos diminuiu em todas as regiões brasileiras (ANDRADE PC, et al., 2004).

As enfermidades recorrentes do processo de envelhecimento já começam a surgir, a partir dos 30 anos trazendo assim complicações para a gestante, feto e recém-nascido, como hipertensão arterial, placenta prévia, diabete gestacional, depressão, baixo peso ao nascer, apgar abaixo de sete no quinto minuto de vida, síndrome de Down entre outros obstáculos puerperais e neonatais, em função disso a gestação tardia deve ser encarada como um importante problema de saúde pública (ZIADEH SM, 2002; ANDRADE PC, et al., 2004).

A quantidade de mulheres que engravidam em idade mais avançada é significativa e configura-se um importante problema de saúde pública, uma vez que tem sido evidenciada associação entre idade materna igual ou superior a 35 anos e resultados perinatais adversos, representados por maior risco de complicações maternas, fetais e no recém-nascido (ZIADEH SM, 2002; ANDRADE PC, et al., 2004).

Em função do exposto, o presente estudo objetivou descrever os desfechos obstétricos das gestações tardias no Estado de Minas Gerais, no período de 2013 a 2017.

\section{MÉTODOS}

Trata-se de estudo transversal descritivo, com utilização dados de natureza secundária oriundos do Sistema de Informação Nascidos Vivos (SINASC) do Departamento de Informática do Sistema Único de Saúde (DATASUS). A população do estudo foi constituída de nascidos vivos, filhos de mães residentes no Estado de Minas Gerais no período de 2013 a 2017.

Os dados foram extraídos do SINASC, fornecido pelo DATASUS, e organizados em arquivo de planilhas do software Microsoft $\circledast$ Office Excel versão 15.0, por dois pesquisadores independentes com o propósito de identificar possíveis discrepâncias. Realizou-se uma análise exploratória para reconhecimento das variáveis e correção de possíveis erros ou inconsistências na digitação dos dados. Feitas as correções necessárias precedeu-se a organização e análise dos dados, aplicando-se a estatística descritiva.

O presente estudo envolveu apenas a descrição e análise de dados secundários de população e nenhuma informação com identificação individual foi obtida para a realização deste estudo. Por se tratar de utilização de dados secundários não houve necessidade de aprovação do comitê de ética.

\section{RESULTADOS}

A amostra do presente estudo foi composta por um total de 1.108 .615 nascidos vivos residentes no Estado de Minas Gerais, cadastrados no SINAN, no período 2013 a 2017, dos quais 192.364 (17,4\%) nasceram de mulheres com idade igual ou superior a 35 anos e $916.251(82,6 \%)$ de mulheres com idade até 34 anos. O perfil demográfico da população do estudo está apresentado na Tabela 1.

A Tabela 2 apresenta duração os desfechos obstétricos, analisando duração da gestação, número de consultas pré-natal, via de parto, índice de Apgar no $1^{\circ}$ e $5^{\circ}$ minuto, peso ao nascer e anomalias congênitas.

A análise dos desfechos obstétricos na gestação tardia demonstrou maior ocorrência de gestação prétermo em 12,9\% RP $=1,25$ (IC95\% 1,23-1,26), com número consultas pré-natal de 7 ou mais consultas $78,6 \% \mathrm{RP}=1,04$ (IC95\% 1,04-1,04), parto cesáreo 70,0\% RP=1,20 (IC95\% 1,19-1,20), índice de Apgar até 7 no $1^{\circ}$ minuto $11,6 \% R P=1,08$ (IC95\% 1,07-1,10), e no 5 minuto $2,3 \% R P=1,20$ (IC95\% 1,16-1,24), baixo peso ao nascer $11,1 \% \mathrm{RP}=1,28$ (IC95\% 1,26-1,29) seguido por alto peso 4,4\% $\mathrm{RP}=1,14$ (IC95\% 1,11$1,17)$ e anomalias congênitas $0,9 \% \mathrm{RP}=1,40$ (IC95\% 1,33-1,48), ambas com associação estatística significativa. 
Tabela 1 - Perfil sociodemográfico dos nascimentos ocorridos no estado de Minas Gerais, no período de 2013 a 2017.

\begin{tabular}{|c|c|c|c|c|}
\hline Variáveis & $\begin{array}{c}\geq 35 \text { anos } \\
n(\%) \text { IC } 95 \%\end{array}$ & $\begin{array}{c}\leq 34 \text { anos } \\
n(\%) \text { IC } 95 \%\end{array}$ & $\begin{array}{c}\text { RP } \\
\text { (IC 95\%) }\end{array}$ & $\mathbf{p}$ \\
\hline Amostra (1.108.615) & $192.364(17,4) 17,3-17,4$ & $916.251(82,6) 82,6-82,7$ & & \\
\hline \multicolumn{5}{|l|}{ Instrução da mãe } \\
\hline Nenhuma & $1.694(0,9) 0,8-0,9$ & $2.007(0,2) 0,2-0,2$ & $4,02(3,77-4,29)$ & $<0,001$ \\
\hline Até 7 anos & $42.236(22,0) 21,8-22,1)$ & $151.897(16,6) 16,5-16,7$ & $1,32(1,31-1,34)$ & $<0,001$ \\
\hline 8 anos ou + & $144.204(75,0) 74,8-75,2$ & $739.922(80,8) 80,7-80,8$ & $0,93(0,93-0,93)$ & $<0,001$ \\
\hline Ignorado & $4.230(2,2) 2,1-2,3$ & $22.425(2,4) 2,4-2,5$ & $0,90(0,87-0,93)$ & $<0,001$ \\
\hline \multicolumn{5}{|l|}{ Estado civil da mãe } \\
\hline Sem companheiro & $53.724(27,9) 27,7-28,1)$ & $346.073(37,8) 37,7-37,9$ & $0,74(0,73-0,75)$ & $<0,001$ \\
\hline Com companheiro & $136.569(71,0) 70,8-71,2$ & $559.713(61,1) 61,0-61,2$ & $1,16(1,16-1,17)$ & $<0,001$ \\
\hline Ignorado & $2.071(1,1) 1,0-1,1$ & $10.465(1,1) 1,1-1,2$ & $0,94(0,90-0,99)$ & 0,013 \\
\hline \multicolumn{5}{|c|}{ Raça / cor do recém nascido } \\
\hline Branca & $77.202(40,1) 39,9-40,4$ & $307.673(33,6) 33,5-33,7$ & $1,20(1,19-1,20)$ & $<0,001$ \\
\hline Preta/Parda & $101.727(52,9) 52,7-53,1$ & $540.950(59,0) 58,9-59,1$ & $0,90(0,89-0,90)$ & $<0,001$ \\
\hline Amarela/Indígena & $1.294(0,7) 0,6-0,7$ & $7.307(0,8) 0,8-0,8$ & $0,84(0,80-0,89)$ & $<0,001$ \\
\hline Ignorado & $12.141(6,3) 6,2-6,4$ & $60.321(6,6) 6,5-6,6$ & $0,96(0,94-0,98)$ & $<0,001$ \\
\hline
\end{tabular}

Legenda: RP= Razão de Prevalência. Fonte: SINASC/DATASUS, 2019.

REAEnf/EJNC | Vol. 3 | e2555 | DOI: https://doi.org/10.25248/REAenf.e2555.2020 Página 4 de 9 
Tabela 2 - Pré-natal e desfechos obstétricos.

\begin{tabular}{|c|c|c|c|c|}
\hline Variáveis & $\begin{array}{c}\geq 35 \text { anos } \\
n(\%) \text { IC } 95 \%\end{array}$ & $n \begin{array}{l}\leq 34 \text { anos } \\
(\%) \text { IC } 95 \%\end{array}$ & $\begin{array}{c}\mathbf{R P} \\
\text { (IC 95\%) }\end{array}$ & $\mathbf{p}$ \\
\hline Amostra (1.108.615) & $192.364(17,4) 17,3-17,4$ & $916.251(82,6) 82,6-82,7$ & & \\
\hline \multicolumn{5}{|l|}{ Duração da gestação } \\
\hline Pré-termo & $24.886(12,9) 12,8-13,1$ & $95.005(10,4) 10,3-10,4$ & $1,25(1,23-1,26)$ & $<0,001$ \\
\hline À termo & $160.104(83,2) 83,1-83,4$ & $774.533(84,5) 84,5-84,6$ & $0,98(0,98-0,99)$ & $<0,001$ \\
\hline Pós-termo & $3.333(1,7) 1,7-1,8$ & $24.648(2,7) 2,7-2,7$ & $0,64(0,62-0,67)$ & $<0,001$ \\
\hline Ignorado & $4.041(2,1) 2,0-2,2$ & $22.065(2,4) 2,4-2,4$ & $0,87(0,84-0,90)$ & $<0,001$ \\
\hline \multicolumn{5}{|l|}{ Consultas Pré-Natal( $\left.\mathrm{n}^{\circ}\right)$} \\
\hline Até 6 & $40.392(21,0) 20,8-21,2$ & $219.066(23,9) 23,8-24,0$ & $0,88(0,87-0,89)$ & $<0,001$ \\
\hline $7+$ & $151.182(78,6) 78,4-78,8$ & $693.637(75,7) 75,6-75,8$ & $1,04(1,04-1,04$ & $<0,001$ \\
\hline Ignorado & $790(0,4) 0,4-0,4$ & $3.548(0,4) 0,4-0,4$ & $1,06(0,98-1,15)$ & 0,134 \\
\hline \multicolumn{5}{|l|}{ Via de parto } \\
\hline Vaginal & $57.379(29,8) 29,6-30,0$ & $379.322(41,4) 41,3-41,5$ & $0,72(0,72-0,73)$ & $<0,001$ \\
\hline Cesáreo & $134.654(70,0) 69,8-70,2$ & $534.996(58,4) 58,3-58,5$ & $1,20(1,19-1,20)$ & $<0,001$ \\
\hline Ignorado & $331(0,2) 0,2-0,2$ & $1.933(0,2) 0,2-0,2$ & $0,82(0,73-0,92)$ & $<0,001$ \\
\hline \multicolumn{5}{|l|}{ Apgar $1^{\circ}$ minuto } \\
\hline Até 7 & $22.347(11,6) 11,5-11,8$ & $98.358(10,7) 10,7-10,8$ & $1,08(1,07-1,10)$ & $<0,001$ \\
\hline 8 a 10 & $165.494(86,0) 85,9-86,2$ & $790.577(86,3) 86,2-86,4$ & $1,00(1,00-1,00)$ & 0,004 \\
\hline Ignorado & $4.523(2,4) 2,3-2,4$ & $27.316(3,0) 2,9-3,0$ & $0,79(0,76-0,81)$ & $<0,001$ \\
\hline \multicolumn{5}{|l|}{ Apgar $5^{\circ}$ minuto } \\
\hline Até 7 & $4.464(2,3) 2,3-2,4$ & $17.740(1,9) 1,9-2,0$ & $1,20(1,16-1,24)$ & $<0,001$ \\
\hline 8 a 10 & $183.448(95,4) 95,3-95,5$ & $871.743(95,1) 95,1-95,2$ & $1,00(1,00-1,00)$ & $<0,001$ \\
\hline lgnorado & $4.452(2,3) 2,2-2,4$ & $26.768(2,9) 2,9-3,0$ & $0,79(0,77-0,82$ & $<0,001$ \\
\hline \multicolumn{5}{|l|}{ Peso ao nascer(g) } \\
\hline Baixo peso (<2500) & $21.326(11,1) 10,9-11,2$ & $79.618(8,7) 8,6-8,7$ & $1,28(1,26-1,29)$ & $<0,001$ \\
\hline Peso normal (2500 a 3999) & $162.529(84,5) 84,3-84,7$ & $801.125(87,4) 87,4-87,5$ & $0,97(0,96-0,97)$ & $<0,001$ \\
\hline Alto peso $(4000+)$ & $8.475(4,4) 4,3-4,5$ & $35.426(3,9) 3,8-3,9$ & $1,14(1,11-1,17)$ & $<0,001$ \\
\hline Ignorado & $34(0,0) 0,0-0,0$ & $82(0,0) 0,0-0,0$ & $1,97(1,32-2,95)$ & $<0,001$ \\
\hline \multicolumn{5}{|l|}{ Anomalias congênitas } \\
\hline Sim & $1.709(0,9) 0,8-0,9$ & $5.796(0,6) 0,6-0,6$ & $1,40(1,33-1,48)$ & $<0,001$ \\
\hline Não & $183.932(95,6) 95,5-95,7$ & $876.193(95,6) 95,6-95,7$ & $1,00(1,00-1,00)$ & 0,824 \\
\hline Ignorado & $6.723(3,5) 3,4-3,6$ & $34.262(3,7) 3,7-3,8$ & $0,93(0,91-0,96)$ & $<0,001$ \\
\hline
\end{tabular}

REAEnf/EJNC | Vol. 3 | e2555 | DOI: https://doi.org/10.25248/REAenf.e2555.2020 Página 5 de 9 


\section{DISCUSSÃO}

As características demográficas analisadas demonstraram maiores proporções de gestação tardias entre mulheres analfabetas $(R P=4,02)$ e com até sete $(7)$ anos de estudo $(R P=1,32)$; com companheiro $(R P=1,16)$ e entre mulheres brancas $(R P=1,20)$.

Esses resultados são divergentes de pesquisa realizada nos Estados Unidos com 36.056 mulheres, que verificou maior escolaridade entre as mulheres acima de 35 anos (CLEARY-GOLDMAN J, et al., 2005). A maior proporção de analfabetismo e baixa escolaridade encontrada no presente estudo pode estar associada à condição socioeconômica desfavorável. $O$ analfabetismo e a baixa escolaridade têm sido associados a desfechos obstétricos desfavoráveis e a mortalidade materna (LAURENTI R, et al., 2004; LEAL MG, et al., 2019). No entanto, são múltiplos os fatores sociais, educacionais, econômicos e culturais que fazem com que as mulheres protelem a gestação (GRAVENA AA et al., 2012).

Estudo utilizando dados secundários do Sistema de Informação Nascidos Vivos de mães residentes no Estado de Minas Gerais no período de 2013 a 2017, ao analisar os percentuais brutos, estes, vão de encontro com nossos achados, confirmando que em sua maioria as mães com mais de 35 anos são pardas ou pretas (ALENCAR NPFC, et al., 2020). Entretanto, foi possível observar que a gestação tardia está estatisticamente associada a raça/cor branca $\mathrm{RP}=1,20$ (IC95\% 1,19-1,20).

Os critérios étnicos-raciais têm sido relacionados à desigualdades na assistência à saúde, onde pretos, pardos e indígenas, apresentam níveis mais elevados de adoecimento e de morte por causas evitáveis, maiores dificuldades de acesso ao serviços de saúde e sua utilização, incluído acesso ao pré-natal, desfechos obstétricos desfavoráveis e maiores índices de mortalidade materna, independente da idade (LOPES F, 2005; DOMINGUES RMSM, et al., 2015).

Quanto à situação conjugal, observou-se que a maioria das gestantes acima de 35 anos viviam com companheiro, todavia, é importante salientar que este fato pode não significar apoio familiar adequado. $O$ fato da mulher viver com companheiro tem sido associado à melhor evolução da gestação e que a falta de vínculo e apoio social, representa um importante fator de risco relacionado a desfechos obstétricos e perinatais desfavoráveis (LEITE RMB, et al., 2011).

Apesar de conhecido os resultados positivos da participação do homem em todo o processo de gestação nossa sociedade ainda segue valores de uma cultura patriarcal e machista que mantem esse período como próprio da mulher. Uma forma de amenizar os impactos que isso pode causar é estimular a participação do parceiro com atitudes como inclui-lo efetivamente nas consultas de pré-natal e no trabalho de parto, colocar seu nome na placa de identificação do recém-nascido, dentre outras atitudes que são propostas pelo Ministério da Saúde (BRASIL, 2018).

No que diz respeito aos desfechos obstétricos, maioria de partos pré termos foi observado nas gestações tardias $\mathrm{RP}=1,25$ (IC95\% 1,23-1,26) com associação estatística significativa. Esses achados são semelhantes a um estudo realizado no Líbano, que demonstrou que as proporções de partos pré-termo entre aquelas com mais idade foi significativamente mais altas, ainda que excluídas as mulheres em que a indução do parto foi indicada pela equipe de saúde, o que reflete o risco de partos pré-termo nesta população. (SEOUD MA, et al., 2002). Outro estudo realizado em Taiwan, também constatou que mulheres com idade maior que 35 anos apresentam maior risco de parto prematuro (HSIEH TT, et al., 2010).

Como componente da atenção integral à saúde materno-infantil, no âmbito do Sistema Único de Saúde (SUS), a assistência pré-natal corresponde a toda assistência, acompanhamento, auxílio e orientação oferecidos às gestantes durante gestação, bem como o apoio psicológico, exames, rastreamento e medidas preventivas das intercorrências maternas no parto e nos desfechos neonatais. Sendo considerado adequado o mínimo de seis consultas durante a gestação e a garantia de todos os exames e medidas preventivas (BRASIL, 2018). Entre as mulheres maiores de 35 anos a adequação ao número de consultas pré-natal foi ligeiramente maior, com 7 ou mais consultas $R P=1,04$ (IC95\% 1,04-1,04), com associação estatística significativa. Tais achados são convergentes com estudo realizado no Paraná, que demonstrou maior adequação de consultas pré-natal em mulheres maiores de 35 anos e associação de número de 
consultas menor que 7 com desfechos obstétricos desfavoráveis, incluindo baixo peso ao nascer (SASS A, et al., 2011).

Com relação à via de parto, maior proporção de cesarianas ocorreu em mulheres de idade avançada $\mathrm{RP}=1,20$ (IC95\% 1,19-1,20), com associação estatística significativa. Em Taiwan, achados semelhantes foram encontrados em estudo, com maioria de cesarianas entre mulheres acima de 35 anos, vale ressaltar ainda que as complicações relacionadas ao parto cesáreo foram de 1,6 a 2,6 vezes maiores nessa faixa etária (HSIEH TT, et al., 2010).

A cesariana é um procedimento cirúrgico, que está associado a risco e complicações tanto para a mãe quanto para o recém nascido, não devendo ser considerada uma alternativa igual ao parto natural, devendo ser respeitada suas indicações. A idade não é por si só, uma indicação para cesariana; pelo contrário, é a ocorrência de riscos específicos nessa faixa etária é que pode levar ou não a uma indicação de parto cesáreo (MYLONAS I e FRIESE K, 2015).

O parto cesariano pode ser indicado em consequência da deterioração da função miometral consequente à idade, que aumenta os riscos de hemorragia pós-parto por atonia uterina, nos casos de apresentação pélvica (principalmente se multípara), desproporção céfalo-pélvica, eclampsia, crise hipertensiva, descolamento prematuro de placenta, e em alguns casos sob indicação fetal como gamelaridade e má-formação congênita (SOUZA ASR, et al., 2010).

Porém, é acompanhado de desvantagens como ocorrência de embolia por líquido amniótico, necessidade do uso profilático de heparina de baixo peso molecular e/ou meias compressivas pelo risco trombótico, infecção cirúrgica, prematuridade e admissão do recém-nascido em UTI neonatal. Portanto, na ausência de fatores de risco o parto normal continua sendo o mais indicado devido à rápida recuperação e à maior certeza de maturidade fetal (SOUZA ASR, et al., 2010).

Baixo índice de Apgar, tanto no primeiro minuto de vida do RN quanto no quinto minuto, foram mais frequentes em gestações tardias, na população do presente estudo, com índice até 7 no $1^{\circ}$ minuto $\mathrm{RP}=1,08$ (IC95\% 1,07-1,10), e no 5 minuto $R P=1,20$ (IC95\% 1,16-1,24), com associação estatística significativa. Um estudo realizado com gestantes de 29 países demonstrou resultado semelhante e risco de índice de Apgar até 7 em gestantes com idade superior a 35 anos (LAOPAIBOON M, et al., 2014).

Os dados também convergem com os encontrados em pesquisa realizada no estado do Maranhão, em um Hospital Universitário que demonstrou maior risco de Apgar inferior a 7 na gestação tardia. (SANTOS GHN, et al., 2009).

Diversos fatores que podem influenciar o índice de Apgar, incluindo sedação ou anestesia, malformações congênitas, idade gestacional, peso ao nascer, pré-eclampsia, trabalho de parto prolongado e está associado ao aumento do risco de desenvolvimento de condições neurológicas como paralisia cerebral, epilepsia e alterações cognitivas (THAVARAJAH H, et al., 2017).

Em relação ao peso ao nascer, maior ocorrência de baixo peso foi observada em mulheres mais velhas $\mathrm{RP}=1,28$ (IC95\% 1,26-1,29), bem como o alto peso $\mathrm{RP}=1,14$ (IC95\% 1,11-1,17). Uma pesquisa retrospectiva que analisou os registros de 9.506 nascimentos no Hospital da Mulher de Liverpool também demonstrou resultados semelhantes (DELPISHEH A et al., 2008).

O baixo peso ao nascer está diretamente associado á mortalidade neonatal em todos os nascimentos. Alguns fatores de risco estão ligados diretamente em associação ao baixo peso ao nascer, mulheres em idade avançada para gestação como, hipertensão, depressão, diabetes, câncer entre outros, esses fatores devem ser acompanhados de perto durante toda a gestação (GRAVENA AA, et.al., 2012).

Maior ocorrência de anomalias congênitas também foi observada nas gestações tardias $\mathrm{RP}=1,40$ (IC95\% 1,33-1,48), convergindo com resultado de estudo realizado em países europeus, que também demonstrou maior proporção de anomalias congênitas como desfecho obstétrico de gestações em mulheres maiores de 35 anos de idade, tendo alterações cromossômicas como achado mais freqüente (CUNHA J, et al., 2002). Todavia, uma pesquisa realizada no Brasil, não demonstrou associação entre idade materna 
avançada e maior ocorrência de anomalia congênita, divergindo de nossos achados (MAGALHÃES MLC, et al., 2006).

Estudo realizado no Paraná avaliou que a gestação em idade avançada pode acontecer de forma não planejada e ser acompanhada de sentimentos ambivalentes como sensação de mais maturidade e alegria pelo momento versus o medo, tanto relacionados aos riscos envolvidos na perda do bebê e ao momento do parto, quanto à própria saúde materna, como mais cansaço, dores, edemas e dificuldade em realizar as atividades do cotidiano. Observa-se que esses medos se fundamentam nos desfechos encontrados nesta pesquisa revelando a necessidade do acompanhamento multiprofissional a essas pacientes, com suporte por parte do médico, enfermeiro, psicólogo e fisioterapeuta, além do incentivo a um suporte familiar adequado a esta gestante (ALDRIGHI JD, et al., 2018).

\section{CONCLUSÃO}

O presente estudo evidenciou maior ocorrência de gestação tardia em mulheres brancas, analfabetas ou com baixa escolaridade, vivendo com companheiro. Desfechos obstétricos desfavoráveis como prematuridade, parto cesáreo, índice de Apgar no primeiro e quinto minutos, baixo peso e alto peso ao nascer e anomalias congênitas também foram mais frequentes nessa população. Entretanto, número de consultas de pré-natal maior ou igual a sete foi mais freqüente entre as mulheres maiores de 35 anos.

\section{REFERÊNCIAS}

1. ALDRIGHI JD, et al. Vivência de mulheres na gestação em idade tardia. Rev Gaúcha Enferm. 2018; 39:e20170112.

2. ALENCAR NPFC. Perfil de nascimentos no estado de Minas Gerais no período de 2013 a 2017. REAEnf/EJNC. 2020; 2; e2354: 1-7.

3. ANDRADE PC, et al. Resultados perinatais em grávidas com mais de 35 anos: estudo controlado. Rev Bras Ginecol Obstet. 2004; 26:697-702.

4. BRASIL. Ministério da Saúde, Secretaria de Atenção à Saúde. Departamento de Ações Programáticas Estratégicas.Guia do pré-natal do parceiro para profissionais de Saúde /, Secretaria de Atenção à Saúde. Brasília: Ministério da Saúde, 2018.

5. CLEARY-GOLDMAN J, et al. Impact of Maternal Age on Obstetric Outcome. Obstet Gynecol., 2005; $105(5)$ : $983-90$.

6. CUNHA J, et al. Defeitos congênitos em Porto Alegre: uma investigação da qualidade dos dados registrados na Declaração de Nascido Vivo. Rev. bras. epidemiol., 2002; 5(suppl.1): 51.

7. DELPISHEH A, et al. Pregnancy late inlife: a hospital-based study of birth outcomes. J Womens Health, 2008; 17(6): 965-70.

8. DOMINGUES RMSM, et al. Adequação da assistência pré-natal segundo as características maternas no Brasil. Rev Panam Salud Pública, 2015; 37(3): 140-7.

9. GRAVENA AA, et al. Outcomes in late-age pregnancies. Rev Esc Enferm USP. 2012; 46(1): $15-21$.

10. HSIEH TT, et al. Advanced maternal age and adverse perinatal outcomes in an Asian population. Eur J Obstet Gynecol Reprod Biol, 2010; 148(1): 21-6.

11. LAOPAIBOON M, et al. Advanced maternal age and pregnancy outcomes: a multicountry assessment. BJOG, 2014; 121(Suppl. 1): 49-56.

12. LAURENTI R, et al. A mortalidade materna nas capitais brasileiras: algumas características e estimativa de um fator de ajuste. Rev Bras Epidemiol, 2004; 7:449-60.

13. LEAL MG, et al. Abortamento no brasil: características sócio-demográficas e fatores condicionantes aos riscos associados com a mortalidade materna. Revista Uningá, 2019; 56(2): 255-267.

14. LEITE RMB, et al. Fatores de risco para mortalidade materna em área urbana do nordeste do Brasil. Cad Saúde Pública., 2011; 27(10): 1977-85.

15. LOPES F. Para além da barreira dos números: desigualdades raciais e saúde. Cad Saúde Pública, 2005; 21(5): 1595-601.

16. MAGALHÃES MLC, et al. Gestação na adolescência precoce e tardia: há diferença nos riscos obstétricos? Rev. Bras. Ginecol. Obstet., 2006; 28(8): 446-452.

17. MALDONADO, M. T. Psicologia da gravidez: parto e puerpério.16ed. São Paulo: Saraiva, 2002; 229p. 
18. MARTIN JA, et al. Annual summary of vital statistics: 2006. Pediatrics, 2008; 121: 788-801.

19. MATHEWS TJ, HAMILTON BE. Idade média da mãe, 1970-2000. Natl Vital Stat Rep., 2002; 51: 1-13.

20. MYLONAS I, FRIESE K. Indications for and Risks of Elective Cesarean Section. Dtsch Arztebl Int., 2015; 112(2930): 489-495.

21. SANTOS GHN, et al. Impactoda idade materna sobre os resultados perinatais e via departo. Rev Bras Ginecol Obstet., 2009; 31(7): 326-34.

22. SASS A, et al. Resultados perinatais nos extremos da vida reprodutiva e fatores associados ao baixo peso ao nascer. Rev. Gaúcha. Enferm., 2011; 32(2): 352-8.

23. SENESI LG, et al. Morbidade e mortalidade neonatais relacionadas à idade materna igual ou superior a 35 anos, segundo a paridade. Rev Bras Ginecol Obstet., 2004; 26(6): 477-82.

24. SEOUD MA, et al. Impact of advanced maternal age on pregnancy outcomes.Am J Perinatol., 2002; $19(1): 1-8$.

25. SOUZA ASR, et al. Indicações de cesariana baseada em evidências: parte II. FEMINA. 2010; $38: 9$.

26. TANAKA ACA, ALVARENGA AT. Tecnologias e medicalização na concepção e anticoncepção. In Saúde sexual e reprodutiva no Brasil. Hucitec, [S.I: s.n.], 1999; 198-208.

27. TANBO T. A fertilidade da população mudou? Tidsskr Nor Laegeforen, 2008; 128: 330-333.

28. THAVARAJAH H, et al. The relationship between the five-minute Apgar score, mode of birth and neonatal outcomes. The Journal of Maternal-Fetal \& Neonatal Medicine, 2017; 31(10): 1335-1341.

29. TSUNECHIRO MA, BONADIO IC. A família na rede de apoio da gestante. Fam Saúde Desenvol, 1999; 1(1/2): 1036.

30. ZIADEH SM. Maternal and perinatal outcome in nulliparous women aged 35 and older. Gynecol Obstet Invest., 2002; 54(1): 6-10. 